Fabrication of nanoscale zero-mode waveguides using microlithography for single molecule sensing

This article has been downloaded from IOPscience. Please scroll down to see the full text article.

2012 Nanotechnology 23455301

(http://iopscience.iop.org/0957-4484/23/45/455301)

View the table of contents for this issue, or go to the journal homepage for more

Download details:

IP Address: 141.211.173.82

The article was downloaded on 25/06/2013 at 20:31

Please note that terms and conditions apply. 


\title{
Fabrication of nanoscale zero-mode waveguides using microlithography for single molecule sensing
}

\author{
Chu-Hsiang Teng ${ }^{1,3}$, Troy A Lionberger ${ }^{2,3}$, Jin Zhang ${ }^{1}$, Edgar Meyhöfer ${ }^{2}$ \\ and Pei-Cheng $\mathrm{Ku}^{1}$ \\ ${ }^{1}$ Department of Electrical Engineering and Computer Science, University of Michigan, 1301 Beal \\ Avenue, Ann Arbor, MI 48105, USA \\ ${ }^{2}$ Department of Mechanical Engineering, University of Michigan, 2350 Hayward Street, Ann Arbor, \\ MI 48105, USA \\ E-mail: meyhofer@umich.edu and peicheng@umich.edu
}

Received 14 May 2012, in final form 12 July 2012

Published 19 October 2012

Online at stacks.iop.org/Nano/23/455301

\begin{abstract}
We present a novel approach to the fabrication of zero-mode waveguides (ZMWs) using inexpensive processing techniques. Our method is capable of rapid fabrication of circular nanoapertures with diameters ranging from $70 \mathrm{~nm}$ to $2 \mu \mathrm{m}$, allowing us to perform a detailed characterization of the dependence of the fluorescence emission on the waveguide diameter. We also validated the use of the fabricated ZMWs by detecting single molecule binding events with a signal-to-noise ratio of ten.
\end{abstract}

(Some figures may appear in colour only in the online journal)

\section{Introduction}

Zero-mode waveguides (ZMWs) are nanoscale metal apertures within which no propagating modes of light can exist. The optical intensity of light incident at a $\mathrm{ZMW}$ decays exponentially as a function of the depth, resulting in a zeptoliter-scale excitation volume confined near the entrance of the waveguide [1]. Consequently, ZMWs are a powerful tool particularly for biophysicists, and make possible for the first time the detection of single biomolecules at physiologically relevant concentrations in the range of 1-100 micromolar [2, 3]. In recent years, ZMWs have enabled studies of real-time DNA sequencing [2, 4, 5], protein-protein interactions [6, 7], translation dynamics of mRNA [8, 9], protein oligomerization [10] and lipid membranes [11-13]. These nanoscopic apertures have also been directly applied to the study of single molecule dynamics in living cell membranes [14]. Importantly, ZMWs require only equipment and instrumentation that is commonly available in many biology laboratories, thus making single molecule experimentation

3 Chu-Hsiang Teng and Troy A Lionberger contributed equally to this work. more widely accessible to the broader research community. However, the technology remains beyond the reach of most laboratories because fabrication of the devices themselves remains highly specialized and expensive.

To date, ZMWs have been fabricated using various advanced patterning techniques, including focused-ion beam $[15,16]$, electron-beam (e-beam) lithography [2, 17], deep-UV lithography [17] and nanoimprint lithography [18]. These nanolithographic techniques are either expensive, inherently limited to low throughput, or incompatible with the production of ZMWs with a wide range of dimensions and geometries. In contrast, microscale patterning using microlithography (e.g., i-line photolithography, interference lithography and direct laser writing) is extremely well developed in the semiconductor industry. Compared to nanolithography, patterning processes based on conventional microlithography are usually more cost-effective, reliable and scalable to large-area substrates. However, the relatively limited resolution of microlithography prohibits its direct applicability to the production of ZMWs requiring dimensions significantly below the diffraction limit of visible light. In this paper, a simple aperture shrinking technique using 

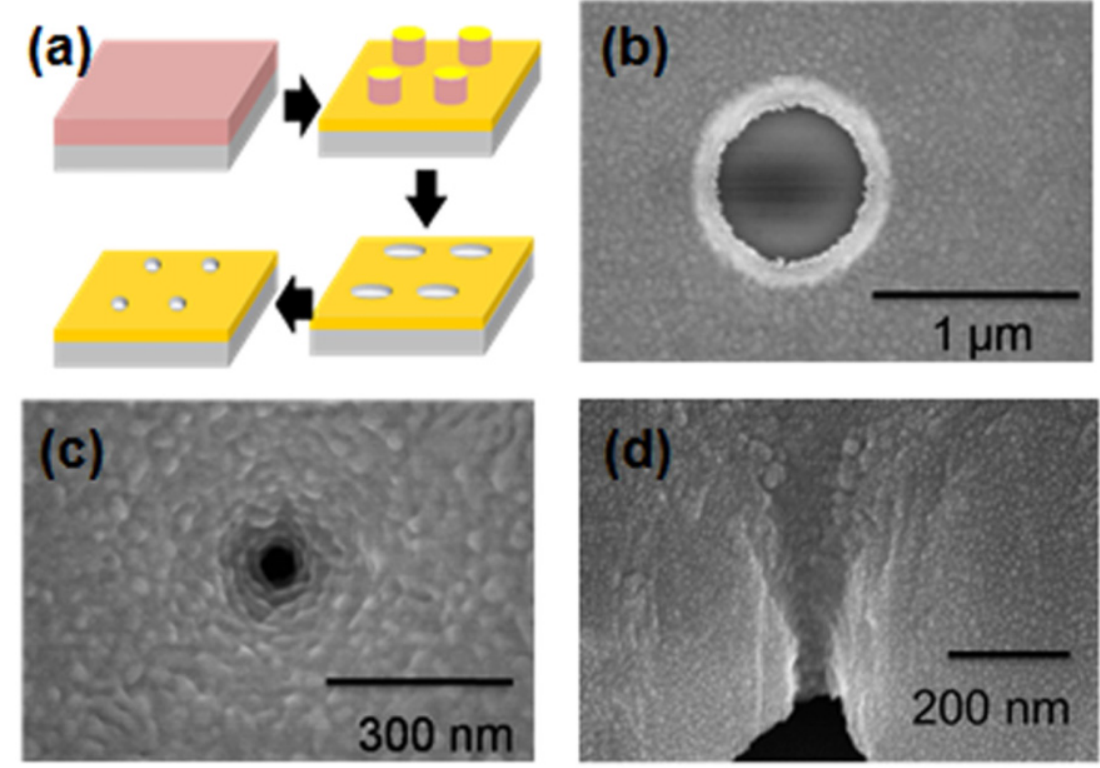

Figure 1. (a) Illustration of the ZMW fabrication process (in clockwise order): photoresist coating; photolithography and gold deposition; lift-off; electrodeposition. (b) Scanning electron microscopy image of a micro-aperture after lift-off. The diameter is $770 \mathrm{~nm}$. (c) SEM image of a ZMW after electrodeposition. The diameter of this waveguide is $70 \mathrm{~nm}$. (d) Cross-section SEM image of a ZMW taken at a $45^{\circ}$ viewing angle. The darker region at the bottom is the glass substrate. The gold protrusions at the bottom left and right corners of the waveguide are likely resulted from the cleavage of the sample. The sample for imaging was coated with a thin layer $(\sim 10 \mathrm{~nm})$ of gold-palladium alloy to eliminate the charging effect on the glass surface.

electrodeposition was developed to overcome this limitation [19]. We demonstrated the versatility of our method by fabricating metal apertures with a large range of aperture diameters. These devices allowed us to characterize the optical properties of waveguides from the ZMW regime to above the zero-mode cut-off limit and develop an (or further our) understanding of how light behaves in these structures over the entire size range. To demonstrate the viability of the proposed fabrication process to yield devices suitable for single molecule detection, we also showed that fluorescence signals from single molecules can be detected with a signal quality that is comparable to previously published data from ZMWs fabricated using nanolithographic techniques.

\section{Experimental details}

The principle of the proposed ZMW fabrication is illustrated in figure 1(a) and involves only two steps. First, an array of micrometer-scale metallic apertures (micro-apertures) is fabricated using conventional photolithography, metal deposition and lift-off on a large-area glass substrate. Next, these micro-apertures are shrunk to the desired ZMW dimensions using electrodeposition. In this study, two samples, samples A and B, were fabricated. Sample A was made for characterization of nanoapertures, and sample B was aimed at micro-apertures for comparison. Glass substrates (Ted Pella) with areas of $76 \mathrm{~mm} \times 83 \mathrm{~mm}$ and thicknesses of $190-250 \mu \mathrm{m}$ were used. The substrates were first cleaned and spin-coated with a negative resist (MicroChemicals AZ 5214; $1.4 \mu \mathrm{m}$ thickness). An i-line stepper (GCA AS200 AutoStep) was used to pattern the micro-aperture arrays.
Each array had $60 \times 60$ micro-apertures with the same diameter. Aperture diameters varied from $770 \mathrm{~nm}$ to $2 \mu \mathrm{m}$. The spacing between two adjacent waveguides was fixed at $5 \mu \mathrm{m}$ for convenient imaging using conventional fluorescence microscopy. After lithography, a gold film of $100 \mathrm{~nm}$ in thickness was deposited by an e-beam evaporator (EnerJet) and the micro-apertures were formed by lift-off. Only sample A underwent a 2.5 min electrodeposition process using the BDT-510 gold plating solution and achieved nanoapertures. The details of the electrodeposition process can be found in [19]. The current density during the electrodeposition was controlled at $1.4 \mathrm{~mA} \mathrm{~cm}{ }^{-2}$, corresponding to an aperture shrinking rate of $140 \mathrm{~nm} \mathrm{~min}{ }^{-1}$. Figures 1(b) and (c) show the scanning electron micrographs of a waveguide before and after the electrodeposition. As shown in the figure, the diameter of the micro-aperture was shrunk from 770 to $70 \mathrm{~nm}$ in sample A. The final waveguide depth (i.e., gold thickness) was $450 \mathrm{~nm}$ inferred from the total shrinkage and confirmed by cross-section SEM.

To form the flow chambers for the fluorescence measurements, the ZMW samples were assembled with conventional, $1 \mathrm{~mm}$ thick glass microscope slides by two lines of double sided tape and silicone grease. Solutions were flowed through the assembled flow chamber using gravity flow. A passivation solution containing $0.1-0.2 \mathrm{mg} \mathrm{ml}^{-1}$ casein was introduced into the flow chamber, incubated for $20 \mathrm{~min}$, flushed from the chamber with a rinse buffer, and then the fluorescent solution was introduced immediately before sealing the chamber with melted wax. 


\section{Results and discussion}

\subsection{ZMW geometry}

To characterize the shape of the ZMWs (see figure 1(c) for an example of a fabricated waveguide), we measured the ellipticity (i.e., $|a-b| / a$, where $a$ and $b$ are the lengths of the long and short axes, respectively) of 50 randomly sampled $70 \mathrm{~nm}$ diameter ZMWs by scanning electron microscopy (SEM; Hitachi SU8000). The measured ellipticity ranged from 0 to 0.17 , where 0 corresponds to a perfectly circular aperture. Compared to e-beam lithography [17], DUV lithography [17] and nanoimprint lithography [18], ZMWs fabricated using our technique exhibit a comparable degree of ellipticity. Since there was no apparent ellipticity before the electrodeposition, we attribute the small ellipticity observed in our ZMWs to the finite grain size of the deposited gold film. Grain boundaries are observed in figure 1(c), and the size of the grains is around $20 \mathrm{~nm}$. When the diameter of the ZMWs becomes comparable to or smaller than the grain size, the shape of the ZMWs will be severely influenced by the grain orientation. Smoother gold surfaces and smaller grains are possible by using a more intricate electrodeposition process, such as pulsed electrodeposition and the use of additives [20]. The size uniformity of the as-fabricated ZMWs was also characterized. Before the electrodeposition, the averaged diameter for the $70 \mathrm{~nm}$ diameter ZMW array was $770 \mathrm{~nm}$ and the standard deviation was $29 \mathrm{~nm}$ for a sample size $(N)$ of 10 that was randomly picked. After the electrodeposition, the averaged diameter was $70 \mathrm{~nm}$ and the standard deviation was $20 \mathrm{~nm}$ for a sample size $(N)$ of 50 . No statistically significant difference in the standard deviation of pre- and post-electrodeposition aperture diameters was found. Therefore, the diameter nonuniformity likely originated from the photolithographic process and photomask rather than from the electrodeposition. This observation also supports the use of electrodeposition to faithfully preserve the geometry as the features are reduced in dimension, a finding that has wide-ranging implications beyond the fabrication of ZMWs. It should be noted that while e-beam lithography is capable of generating features with a smaller variation in size [17], it is also significantly more expensive and is limited in patterning large features. In contrast, our method can be easily scaled to large areas with arbitrary geometries. More than $3 \times 10^{6}$ ZMWs with good uniformity were patterned in parallel on the $76 \mathrm{~mm} \times 83 \mathrm{~mm}$ glass substrate.

Imaging of ZMW cross-sections was also carried out by SEM. Figure 1(d) shows the cross-sectional image of a ZMW device taken at a $45^{\circ}$ tilting angle. The sample was cleaved from the back side to avoid destruction of the ZMW structures. As can be seen in figure 1(d), in spite of the irregular ruptured surface of the gold layer, the sidewall is vertical from the bottom to about $170 \mathrm{~nm}$ above the glass substrate and then exhibits a slope at an angle of $65^{\circ}$ with respect to the glass surface. Since the evanescent depth of the excitation field is much smaller than $170 \mathrm{~nm}$, the sloppy part of the sidewall does not affect the characteristics of the ZMWs.

\subsection{ZMW optical properties}

To characterize the optical properties of the ZMWs, we measured the fluorescence emission from the ZMWs using a $10 \mathrm{mM}$ concentration of the fluorescent dye rhodamine B $\left(\lambda_{\mathrm{em}}=625 \mathrm{~nm}\right)$. The dye solution was prepared at a high concentration such that the number of molecules in the waveguide remained approximately constant for all waveguide sizes while individual dye molecules were diffusing in and out of the excitation volume. In this way, we ensured a consistent comparison from different waveguide sizes and, therefore, different excitation volumes. A mercury arc lamp was used as the excitation light source to ensure uniform excitation over the entire field of view by avoiding interference using coherent light sources. A bandpass excitation filter was placed in front of the arc lamp to select a narrow spectral range. The $546 \mathrm{~nm}$ $\left(\lambda_{\mathrm{E}}\right)$ mercury spectral line was used in the experiment. Köhler epi-illumination of the waveguide arrays was achieved using an inverted fluorescence microscope (Zeiss Axiovert 200) to ensure uniform excitation of the ZMWs within the field of view. We measured the fluorescence emission from the nanoapertures with dimensions spanning three different regimes: (1) waveguides with diameters smaller than the zero-mode cut-off of $0.586 \lambda_{\mathrm{E}} / n$ or $240 \mathrm{~nm}$, where $n$ is the refractive index of the dye solution [21] (figure 2); (2) waveguides with optical characteristics between ZMWs and conventional metallic waveguides (figure 3); and (3) waveguides behaving as conventional metallic waveguides (above $1 \mu \mathrm{m}$, see figure 4). Sample A was used to characterize waveguides in regimes (1) and (2), and sample B was for regime (3). The goal of the measurements was to demonstrate that the fluorescence signal from the broad size range of waveguides made by electrodeposition agrees with previously observed properties using different fabrication techniques. Based on the equation of the fluorescence signal from different positions in a waveguide used in [2], the fluorescence intensity $F$ can be described as the integration of fluorescence signal over the volume of a waveguide. Here we assumed that the change of photokinetics in ZMWs is independent of ZMW diameter. This assumption allows us to express $F$ as follows:

$$
F=C \pi\left(\frac{D}{2}\right)^{2} \int_{0}^{t}[I(z) P(z)] \mathrm{d} z .
$$

In (1), $D, t, I$ and $P$ correspond to the waveguide diameter, gold layer thickness, excitation intensity inside the waveguide and fluorescence out-coupling efficiency, respectively. The intensity of fluorescence emission by a dye molecule is assumed to be proportional to the excitation intensity, and the fluorescence out-coupling efficiency represents the fraction of fluorescent signal that can be collected by the detector. Thus $I(z) P(z)$ is proportional to the detected fluorescence intensity from different $z$ positions. The constant $C$ represents the radial dependence of both $I(z)$ and $P(z)$ which should be independent of $D$. This is partly justified by the small penetration depth of light into the waveguide sidewall and partly by the uncertainty in the number and positions of the fluorescent molecules inside the waveguide at any given 


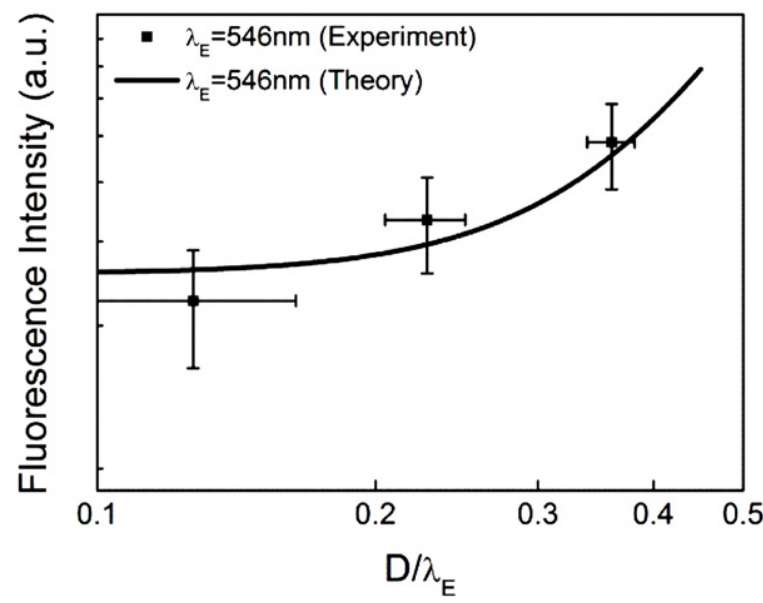

Figure 2. Fluorescence intensity measured from $D=70,124$, and $196 \mathrm{~nm}$ waveguide arrays. These ZMWs are in the zero-mode regime. The diameter is normalized to the excitation wavelength. The theoretical curves are obtained from FDTD simulations.

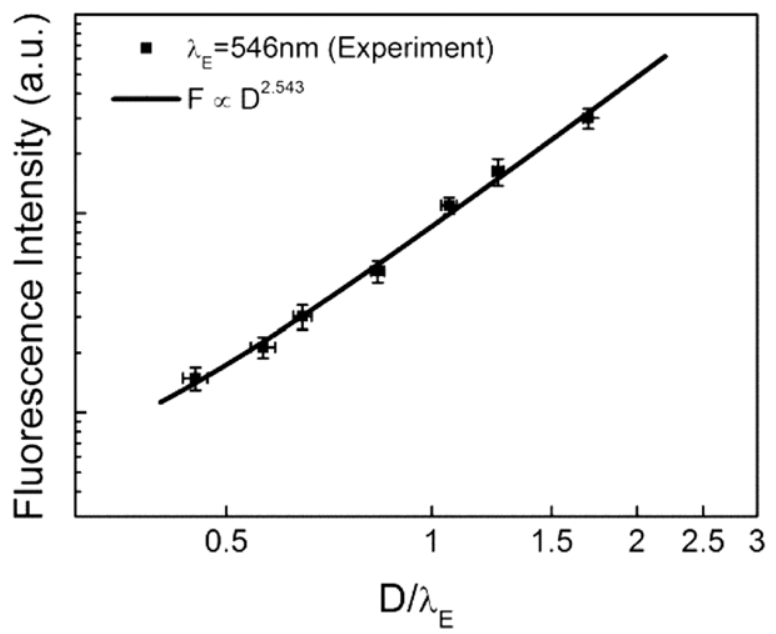

Figure 3. Fluorescence intensity measured from $D=246 \mathrm{~nm}-927 \mathrm{~nm}$. The solid line is the best fit with a power dependence of 2.54 on the waveguide diameter.

time. Hence the fluorescent signal measured was the averaged value across the radial direction of the waveguide. The possible enhancement of quantum yield in a ZMW was assumed to be independent of ZMW diameter and was also incorporated in the constant $C$. It is expected that the fluorescent intensity should increase proportionally with the volume of the excitation. We plotted $F$ as a function of the dimensionless waveguide diameter $\left(D / \lambda_{\mathrm{E}}\right)$ such that the result is applicable to different excitation wavelengths too. The data for waveguide diameters in figures 2 and 3 were measured by SEM from 50 randomly sampled waveguides of the same nominal sizes, while in figure 4the data are from 10 waveguides. The data for the fluorescence signal were averaged over the entire $60 \times 60$ array.

To model $F$ versus $D / \lambda E$ in the $\mathrm{ZMW}$ regime, we numerically calculated (1) using the finite-difference-timedomain (FDTD) method. Since no propagation modes exist in the waveguide, the intensity of light whether

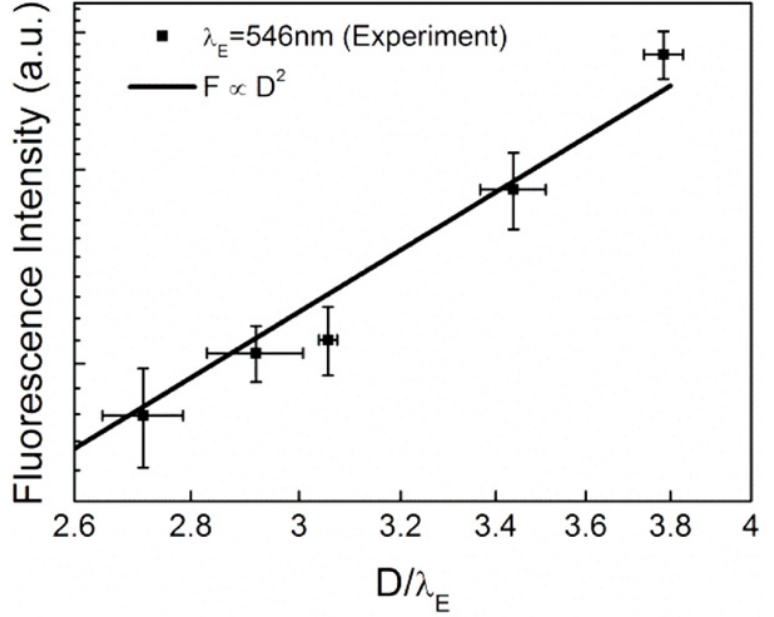

Figure 4. Fluorescence intensity measured from $D>1 \mu \mathrm{m}$. The solid line shows a second power dependence on the waveguide diameter.

incident externally or generated internally should exhibit an exponential dependence on $z$, i.e. $I(z)=\mathrm{e}^{-z / L_{1}}$ and $P(z)=$ $\mathrm{e}^{-z / L_{2}}$. To simplify the numerical model, $L_{1}$ and $L_{2}$ were assumed to have the same dependence on waveguide diameter. The FDTD simulations were carried out to determine the dependence of the characteristic decay length on $D / \lambda_{\mathrm{E}}$. A plane wave CW $546 \mathrm{~nm}$ source was placed at the substrate side. Perfectly matched layers (PMLs) were used at all boundaries. The refractive index dispersion of $\mathrm{Au}$ was obtained from [22]. The simulation results revealed that the decay length has a $\left(D / \lambda_{\mathrm{E}}\right)^{1.41}$ dependence. According to (1), the overall dependence of $F$ now becomes $\left(D / \lambda_{\mathrm{E}}\right)^{3.41}$. This power dependence agreed well with the measurement results (figure 2). It should be noted that any changes in the fluorescence quantum yield owing to the localization within a nanoscale environment were not considered in our analysis [23].

Above the ZMW cut-off, $F$ versus $D / \lambda_{\mathrm{E}}$ did not show a sudden change of its power dependence from the ZMW regime 3.14 to 2 as shown in figures 3 and 4 . The transition is explained by the increase of the number of propagation modes inside the waveguide above the ZMW cut-off. When the waveguide diameter is much greater than the optical wavelength, the number of propagation modes is large and therefore the excitation intensity becomes relatively constant inside the waveguide. As a result, the fluorescent intensity only depends on the area of the nanoaperture as shown in figure 4 . In the transition regime, the number of propagation modes supported within the waveguides increases with the waveguide diameter. The power dependence is between 2 and 3.4 as shown in figure 3 .

It is noted that in all three regimes, the measurements were not affected by the generation of surface plasmons in the gold film because the excitation and fluorescence wavelengths were shorter than the surface plasmon resonance at the gold/water interface [24]. Although the surface plasmonic effect will increase the effective excitation volume and fluorescent quantum yield, the fluorescence intensity can also 
become sensitive to the ZMW diameter [25] and thus a more sophisticated model is required to optimize the ZMW design.

\subsection{Single molecule detection}

As discussed earlier, the fabrication methodology presented here is intended to rapidly and cost-effectively fabricate zero-mode waveguides for single molecule detection assays. To characterize the performance of the nanoapertures for single molecule detection, we observed the diffusion of a $\sim 53 \mathrm{kDa}$ tetramethylrhodamine-labeled streptavidin (TMR-SA, Invitrogen) into and out of the ZMWs. The emission spectrum peaks at $580 \mathrm{~nm}$. At a concentration of $1 \mu \mathrm{M}$, individual fluorescent molecules would not be detectable under bright-field epi-illumination. Even using confocal microscopy, where excitation is confined within a diffraction-limited volume, single diffusing molecules would not be detected above background at micromolar concentrations of fluorescent protein because on average more than 20 molecules would be excited simultaneously. We therefore used a $1 \mu \mathrm{M}$ concentration of TMR-SA to validate the optical confinement of excitation within sub-diffraction limited volumes inside the ZMW apertures. Under bright-field excitation using a collimated $488 \mathrm{~nm}$ argon ion laser (Melles-Griot), single diffusing TMR-SA molecules could be easily detected above background (figure 5, asterisks) by using a Roper Scientific EMCCD camera, 505DCXR dichroic and HQ505LP emission filters. To characterize the quality of the detection signal, we limited the surface passivation to induce nonspecific binding (figure 5, solid bars), which enabled us to quantify the maximum single fluorophore signal (occurring when TMR-SA binds the glass surface at the bottom of the ZMW). We have observed a signal-to-noise ratio as high as 9.7 (figure 5, top trace), where we defined noise as the mean value of peak-to-peak fluctuations and signal as the change of fluorescence intensity when binding events occurred. This demonstrates that the fabricated waveguides are well-suited for single molecule binding assays.

\section{Conclusion}

In summary, a novel ZMW fabrication method is presented. The combination of conventional photolithography, lift-off and electrodeposition provides a cost-effective way of fabricating nanoscale apertures in a metallic film. The proposed method produces ZMWs with good uniformity across a large substrate area with excellent circularity and thus holds a significant potential for mass production and is suitable for bioassays that require a large array of ZMW devices for statistical significance. To demonstrate the versatility of the method, we produced waveguides ranging in size from $70 \mathrm{~nm}$ to over $1 \mu \mathrm{m}$ and characterized the fluorescence signal emitted from the waveguides. Our results show that the dependence of the fluorescence intensity on the waveguide diameter does not increase sharply at the zero-mode cut-off, but instead appears to go through a transition regime until all propagating modes of light are

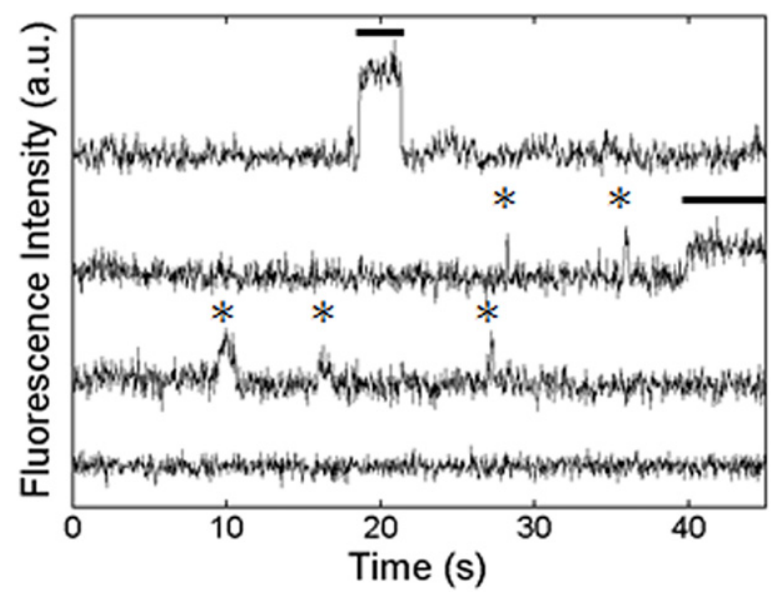

Figure 5. Single molecule detection by time-resolved fluorescence emission from single TMR-labeled streptavidin molecules diffusing into three different nanoapertures of $120 \mathrm{~nm}$ in diameter. Compared to background (bottom trace), diffusion events can clearly be resolved with $40 \mathrm{~ms}$ resolution (asterisks). The surface passivation was limited for the ZMWs used in the measurement in order to introduce nonspecific binding events (solid bars) and reveal the expected maximum signal achievable for single molecule binding assays. Some diffusion events showed lower fluorescence intensity because the molecules did not diffuse to the bottom of the ZMWs and were excited by lower intensity. The traces have been offset for clarity.

represented within the waveguide. We have shown that the power dependence of the fluorescence emission on waveguide diameter gradually varies from 3.4 in the zero-mode regime to 2 when the waveguide diameter is greater than 2.5 times the excitation wavelength. This observation bridges our understanding of how the performance of waveguide apertures varies as a function of diameter and establishes that the background attenuation enabled by nanoapertures does not sharply decline as diameters exceed the cut-off limit. Our results can be applied to fluorescence assays that utilize different waveguide dimensions, excitation wavelength and fluorescence wavelength. Finally, single molecule detection was demonstrated, proving the viability of the proposed fabrication method for single molecule sensing.

\section{Acknowledgments}

This work is partially supported by National Science Foundation grant CBET-0966723 to PCK and National Science Foundation grant CMMI-0969958 to EM.

\section{References}

[1] Betzig E and Trautman J K 1992 Near-field optics: microscopy, spectroscopy, and surface modification beyond the diffraction limit Science 257 189-95

[2] Levene M J, Korlach J, Turner S W, Foquet M, Craighead H G and Webb W W 2003 Zero-mode waveguides for single-molecule analysis at high concentrations Science 299 682-6

[3] Moran-Mirabal J M and Craighead H G 2008 Zero-mode waveguides: sub-wavelength nanostructures for single molecule studies at high concentrations Methods 46 11-7 
[4] Eid J et al 2009 Real-time DNA sequencing from single polymerase molecules Science 323 133-8

[5] Flusberg B A, Webster D R, Lee J H, Travers K J, Olivares E C, Clark T A, Korlach J and Turner S W 2010 Direct detection of DNA methylation during single-molecule, real-time sequencing Nature Methods 7 461-5

[6] Miyake T, Tanii T, Sonobe H, Akahori R, Shimamoto N, Ueno T, Funatsu T and Ohdomari I 2008 Real-time imaging of single-molecule fluorescence with a zero-mode waveguide for the analysis of protein-protein interaction Anal. Chem. 80 6018-22

[7] Sameshima T, Iizuka R, Ueno T, Wada J, Aoki M, Shimamoto N, Ohdomari I, Tanii T and Funatsu T 2010 Single-molecule study on the decay process of the football-shaped GroEL-GroES complex using zero-mode waveguides J. Biol. Chem. 258 23159-64

[8] Petrov A, Kornberg G, O'Leary S, Tsai A, Uemura S and Puglisi J D 2011 Dynamics of the translational machinery Curr. Opin. Struct. Biol. 21 137-45

[9] Uemura S, Aitken C E, Korlach J, Flusberg B A, Turner S W and Puglisi J D 2010 Real-time tRNA transit on single translating ribosomes at codon resolution Nature 464 1012-7

[10] Samiee K T, Foquet M, Guo L, Cox E C and Craighead H G 2005 Lambda-repressor oligomerization kinetics at high concentrations using fluorescence correlation spectroscopy in zero-mode waveguides Biophys. J. 88 2145-53

[11] Kahya N and Schwille P 2006 Fluorescence correlation studies of lipid domains in model membranes (review) Mol. Membr. Biol. 23 29-39

[12] Sako Y and Yanagida T 2003 Single-molecule visualization in cell biology Nature Cell Biol. 4 SS1-5

[13] Samiee K T, Moran-Mirabal J M, Cheung Y K and Craighead H G 2006 Zero mode waveguides for single-molecule spectroscopy on lipid membranes Biophys. J. 90 3288-99

[14] Edel J B, Wu M, Baird B and Craighead H G 2005 High spatial resolution observation of single-molecule dynamics in living cell membranes Biophys. J. 88 L43-5
[15] Rigneault H, Capoulade J, Dintinger J, Wenger J, Bonod N, Popov E, Ebbesen T W and Lenne P-F 2005 Enhancement of single-molecule fluorescence detection in subwavelength apertures Phys. Rev. Lett. 95117401

[16] Gérard D, Wenger J, Bonod N, Popov E, Rigneault H, Mahdavi F, Blair S, Dintinger J and Ebbesen T W 2008 Nanoaperture-enhanced fluorescence: towards higher detection rates with plasmonic metals Phys. Rev. B 77045413

[17] Foquet M, Samiee K T, Kong X, Chauduri B P, Lundquist $\mathrm{P} \mathrm{M}$, Turner $\mathrm{S} \mathrm{W}$, Freudenthal $\mathrm{J}$ and Roitman D B 2008 Improved fabrication of zero-mode waveguides for single-molecule detection J. Appl. Phys. 103034301

[18] Wada J, Ryu S, Asano Y, Ueno T, Funatsu T, Yukawa T, Mizuno J and Tanii T 2011 Fabrication of zero-mode waveguide by ultraviolet nanoimprint lithography lift-off process Japan. J. Appl. Phys. 50 06GK7-GK7-5

[19] Lee L K and Ku P C 2010 Wafer-scale nanopatterning using electrodeposition J. Vac. Sci. Technol. B 28 849-53

[20] Devaraj G, Guruviah S and Seshadri S K 1990 Pulse plating Mater. Chem. Phys. 25 439-61

[21] Keilmann F 1981 Infrared high-pass filter with high contrast Int. J. Infr. Millimeter Waves 2 259-72

[22] Vial A, Grimault A S, Macias D, Barchiesi D and de la Chapelle M L 2005 Improved analytical fit of gold dispersion: application to the modeling of extinction spectra with a finite-difference time-domain method Phys. Rev. B 71085416

[23] Wenger J, Cluzel B, Dintinger J, Bonod N, Fehrembach A L, Popov E, Lenne P F, Ebbesen T W and Rigneault H 2007 Radiative and nonradiative photokinetics alteration inside a single metallic nanometric aperture J. Phys. Chem. C 111 11469-74

[24] Prikulis J, Hanarp P, Olofsson L, Sutherland D and Käll M 2004 Optical spectroscopy of nanometric holes in thin gold films Nano Lett. 4 1003-7

[25] Wenger J, Aouani H, Gerard D, Blair S, Ebbesen T W and Rigneault H 2010 Enhanced fluorescence from metal nanoapertures: physical characterizations and biophotonic applications Plasmonics in Biology and Medicine VII: Proc SPIE ed T Vo-Dinh and J R Lakowicz (Bellingham, WA: Optical Engineering Press) p 75770J 